\title{
HYPERTENSION \\ Dendritic cells: linking oxidation and hypertension
}

The adaptive immune system has a role in the pathogenesis of hypertension, but the mechanisms leading to T cell activation in hypertension are unknown. New findings show that isoketalmodified dendritic cells (DCs), which form in response to oxidative stress, act as neoantigens to promote $\mathrm{T}$ cell proliferation. "The present study establishes a new mechanism of $\mathrm{T}$ cell activation that we believe links oxidation with immune cell activation," explains researcher David Harrison.

Previous studies showing that reactive oxygen species (ROS) contribute to hypertension, together with the finding that spleen and lymph node DCs undergo maturation during angiotensin II-induced hypertension, led Harrison and colleagues to investigate the role of DC NADPH oxidases in the pathogenesis of hypertension. They initially found that angiotensin II increased superoxide levels in DCs, leading to the hypothesis that oxidative and protein modification of DCs might promote $\mathrm{T}$ cell activation. Isoketals are oxidized products of arachidonic acid that react rapidly with protein lysines. Isoketal protein adducts are found in several diseases associated with oxidative stress.

To investigate the potential role of isoketals in hypertension, Harrison and colleagues used a variety of methods to show that DCs accumulate isoketals. Administration of isoketal scavengers prevented DC isoketal accumulation, the onset of hypertension and renal damage in

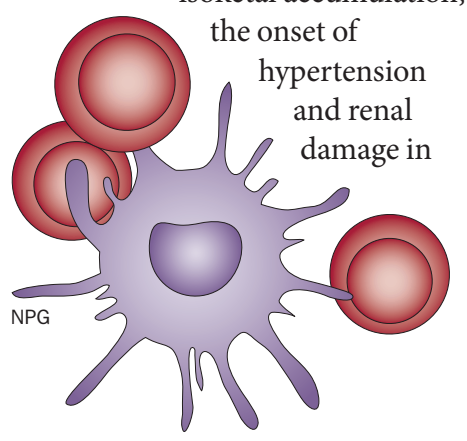

response to angiotensin II. The accumulation of isoketals in DCs was associated with increased DC production of IL-6, IL- $1 \beta$ and IL-23, increased T cell proliferation and increased production of IFN- $\gamma$, IL-17A and TNF by T cells-effects that were all prevented by administration of an isoketal scavenger. The researchers then used adoptive transfer to show that DCs from angiotensin II-treated mice cause a severe hypertension response in recipient mice given a subpressor dose of angiotensin II. Cotreatment of donor mice with an isoketal scavenger prevented this response.

Levels of plasma F2isoprostanes, which are formed together with isoketals, were higher in patients with resistant hypertension than in those with controlled hypertension or normal blood pressure $(23.2 \mathrm{pg} / \mathrm{ml}$ versus $18.3 \mathrm{pg} / \mathrm{ml}$ and $11.6 \mathrm{pg} / \mathrm{ml}$, respectively; $P<0.05)$. Levels of isoketal adducts in monocytes were also higher in patients with hypertension than in those without hypertension. "Our findings are important for several reasons," explains Harrison. "First they provide at least one reason why $\mathrm{T}$ cells begin to produce injurious cytokines in hypertension; second they provide a new link between oxidation and inflammation, which often coexist; finally, they support a previously unknown role for DCs in hypertension."

The researchers are now studying a series of small molecules to determine which is best for scavenging isoketals, in the hope that at least one might be used to control hypertension in humans.

Susan J. Allison
Original article Kirabo, A. et al. DC isoketalmodified proteins activate T cells and promote hypertension. J. Clin. Invest. doi:10.1172/ JCl74084 